\title{
Inflammatory mechanisms linking obesity and metabolic disease
}

\author{
Alan R. Saltiel and Jerrold M. Olefsky \\ Department of Medicine, UCSD, La Jolla, California, USA.
}

\begin{abstract}
There are currently over 1.9 billion people who are obese or overweight, leading to a rise in related health complications, including insulin resistance, type 2 diabetes, cardiovascular disease, liver disease, cancer, and neurodegeneration. The finding that obesity and metabolic disorder are accompanied by chronic low-grade inflammation has fundamentally changed our view of the underlying causes and progression of obesity and metabolic syndrome. We now know that an inflammatory program is activated early in adipose expansion and during chronic obesity, permanently skewing the immune system to a proinflammatory phenotype, and we are beginning to delineate the reciprocal influence of obesity and inflammation. Reviews in this series examine the activation of the innate and adaptive immune system in obesity; inflammation within diabetic islets, brain, liver, gut, and muscle; the role of inflammation in fibrosis and angiogenesis; the factors that contribute to the initiation of inflammation; and therapeutic approaches to modulate inflammation in the context of obesity and metabolic syndrome.
\end{abstract}

We are in the midst of a worldwide obesity epidemic. Over 1.9 billion people are now obese or overweight, including more than 50 million children under age 5 , and the worldwide prevalence of obesity has doubled since 1980 (1). The complications of obesity are taking a major public health toll, which will only worsen in future years. Among the most devastating of these complications is type 2 diabetes (T2D). Most patients with T2D are obese or overweight, and there are numerous longitudinal studies linking obesity with insulin resistance, a frequent forerunner of diabetes.

Over the past two decades, the search for a mechanism linking the pathogenesis of obesity with insulin resistance and diabetes has revealed a close relationship between nutrient excess and activation of the innate immune system in most organs pertinent to energy homeostasis (2-4). Numerous studies indicate that inflammation occurs as a consequence of obesity, and recent insights suggest that it may play a causative role in generating insulin resistance, defective insulin secretion, and disruption of other aspects of energy homeostasis. The nature of obesityinduced inflammation differs from other inflammatory paradigms in that it involves tonic activation of the innate immune system that impacts metabolic homeostasis, in some cases over a lifetime. Inflammation also leads to maladaptive responses such as fibrosis and necrosis that can cause significant tissue damage. Moreover, obesity-induced inflammation is unique in that it involves multiple organs, including adipose, pancreas, liver, skeletal muscle, heart, and brain. These features of obesity-induced inflammation present a challenge to understanding the underlying mechanisms and the manner by which they impact metabolic

Conflict of interest: A.R. Saltiel owns stock in Pfizer Inc. and holds several patents related to treatment of metabolic disease. J.M. Olefsky owns stock in Catabasis Pharmaceuticals and receives consulting income from Cymabay Inc., Second Genome, and AntriaBio. Reference information: / Clin Invest. 2017;127(1):1-4. doi:10.1172/JCI92035. systems. In this issue of the JCI, a group of experts have provided reviews that dive deeply into the mechanisms by which obesity-induced inflammation influences metabolic homeostasis, surveying the modes and mechanisms of inflammation in different tissues and how they might contribute to disease. These Reviews also discuss how inflammation can lead to other aspects of metabolic syndrome, including hyperphagia, reduced energy expenditure, $\beta$ cell dysfunction, and liver disease, and summarize the status of therapeutic interventions that target inflammation in the context of metabolic syndrome.

\section{Activation of the innate immune system in obesity}

Much of what has been learned about the immune response to obesity has come from studies in adipose tissue, although it is clear that inflammation occurs in other organs as well. Adipose depots normally contain multiple immune cells that together surveil and maintain the integrity and hormonal sensitivity of adipocytes. In lean animals, these immune cells operate in an overall Th2 or type 2 state that coordinately controls tissue integrity and metabolism by controlling the activity of subsets of T lymphocytes. These cells release a cascade of cytokines that coordinately regulate other immune cells, including eosinophils, mast cells, and others that maintain resident macrophages in an M2-polarized or alternately activated state (5). In turn, M2-polarized macrophages secrete IL-10 and perhaps other cytokines that contribute to maintenance of insulin sensitivity in adipocytes.

Macrophages undergo dramatic changes during obesity, with an increase in the overall number of macrophages that is largely due to the recruitment of M1-polarized macrophages, which display a more proinflammatory phenotype and secrete cytokines such as TNF- $\alpha$. The increase in the number of macrophages as well as an increased ratio of M1 to M2 macrophages is a hallmark of the adipose tissue inflammation that accompanies obesity, and 
is associated with the development of insulin resistance and metabolic disease (5). Macrophages serve as effectors of a complex immune program triggered by obesity. This program is generally characterized by an overall Th1 or type 1 immune response that is usually activated as a response to infection; however, in the case of obesity this immune reaction initiates a chronic response in adipose tissue that involves a variety of $\mathrm{T}$ effector cells, B cells, NK cells, and others that produce cytokines that govern the accumulation and activity of proinflammatory M1 macrophages (4). Edgar Engleman and colleagues review the roles of innate and adaptive immunity in obesity-related metabolic disease, including the potential role of gut inflammation as an initiating trigger and the molecular mechanisms by which the immune system influences insulin sensitivity (6). Kosei Eguchi and Ryozo Nagai examine the evidence for the role of macrophages in islet inflammation and detail the molecular mechanisms by which inflammatory cytokines contribute to $\beta$ cell dysfunction (7).

The liver is responsible for the majority of glucose production during fasting and is a key site of metabolic homeostasis and inflammation in obesity. Several studies have shown that Kupffer cells, resident hepatic macrophages that play an important role in tissue surveillance in the lean state, can undergo activation of inflammatory pathways during obesity (8). However, obesity also leads to a pronounced increase in recruited hepatic macrophage infiltration, along with the local production of inflammatory chemokines and cytokines that can generate insulin resistance in hepatocytes (9). Similarly, obesity has been associated with the presence of other immune cells in liver, including T cells and neutrophils. Yukinori Koyama and David Brenner discuss the close connection between obesity, diabetes, hepatic inflammation, and the development of nonalcoholic fatty liver disease (NAFLD), which can progress to nonalcoholic steatohepatitis (NASH) and eventually frank cirrhosis due to sequential activation of inflammation and fibrosis (10).

\section{What triggers obesity-induced inflammation?}

The precise triggers of obesity-associated inflammation are poorly understood, and may also differ from tissue to tissue. While obesity is closely associated with increased inflammatory markers in liver, adipose tissue, skeletal muscle, pancreatic islets, and brain, the precise temporal relationships between these events in rodents or obese humans remain uncertain. Kanakadurga Singer and Carey Lumeng review the initiating events in obesity-induced inflammation in the context of human development, many of which are sustained into adulthood (11). Numerous mechanisms have been investigated in rodent models of dietary and genetic obesity $(12,13)$. For example, obesity gives rise to increased intestinal permeability, which results in higher circulating levels of LPS from intestinal Gram-positive bacterial species (14). This gut-derived LPS may initiate an inflammatory cascade via activation of pattern recognition receptors (PRRs) such as TLR4 in fat cells. Similar events are likely to occur in liver. Consistent with this theory, increased circulating LPS is positively correlated with T2D in humans (15). However, intestinalderived LPS is a systemic circulating factor, and so is probably an amplifier of inflammatory processes, rather than a tissue-specific triggering mechanism. Daniel Winer, Tony Lam, and col- leagues described the changes in the intestinal immune system that may influence systemic immunity and glucose metabolism, contributing to the pathology of obesity and diabetes (16).

Different lipid species that are elevated due to diet or obesity may also contribute to inflammation. Free fatty acids can promote inflammation by indirectly binding to TLR 4 and TLR 2 through the adaptor protein fetuin $\mathrm{A}$, resulting in NF- $\mathrm{KB}$ and JNK1 activation (15). Once activated, these pathways can increase the synthesis and secretion of chemokines such as monocyte chemoattractant protein-1 (MCP1) from adipocytes or hepatocytes, leading to infiltration of proinflammatory macrophages. Parenthetically, there are lipid species such as omega- 3 and -6 fatty acids that serve anti-inflammatory roles (17).

There is also evidence that hypoxia develops as adipose tissue expands due to relative underperfusion of the enlarging adipose tissue or increased oxygen consumption (18), and cellular hypoxia may in turn initiate inflammation by inducing the HIF1 gene program. Indeed, genetic deletion of adipocyte HIF1 prevents obesity-induced inflammation and insulin resistance (18). Exposure of adipose tissue in culture to hypoxic conditions can induce upregulation of numerous inflammatory genes (19). Immunostaining of adipose tissue from obese rodents or humans reveals that areas of hypoxia are correlated with regions displaying infiltration of macrophages. Likewise, adipocyte necrosis is closely associated with the development of inflammation.

Another potential mechanism underlying inflammation is mechanical stress on the fat cell. Adipocytes interact with their extracellular matrix (ECM) via pathways that govern differentiation and expansion in response to obesity (20). Expansion of adipocytes in the ECM-fixed environment can elicit various mechanical stresses on these cells. Indeed, adipocyte knockout of collagen genes (21) or the collagenases that degrade collagen, particularly MMP14, has a major impact on lipid synthesis, storage, and energy metabolism (22).

In addition to metabolic dysfunction in the peripheral organs, obesity and obesity-associated inflammation have also been linked to alterations in brain function, particularly in areas that regulate energy homeostasis and systemic metabolism. The hypothalamus controls neuroendocrine circuits, including the melanocortin system, that regulate feeding behavior and energy expenditure. High-fat/high-calorie diets have been shown to induce inflammatory processes in the hypothalamus prior to the induction of these events in the peripheral tissues. Alexander Jais and Jens Brüning describe the contributions of different neuronal and non-neuronal cell types to hypothalamic inflammation (23).

\section{Mechanisms of inflammation-induced insulin resistance}

There is now overwhelming evidence that inflammation is directly associated with insulin resistance. Inhibition of inflammatory signaling by knockout of key pathways in obese mice, including components of NF- $\mathrm{KB}$ (24) and JNK (25) pathways, as well as numerous other proinflammatory signaling molecules, scaffolding proteins, and cytokines, can disrupt the link between obesity and insulin resistance $(15,26-33)$. Many of these pathways can directly or indirectly block insulin action. The arachidonic acidderived product leukotriene B4 (LTB4) is released from adipo- 
cytes and attracts macrophages to adipose tissue and may also directly attenuate insulin signaling in myocytes and hepatocytes (34). Likewise, galectin-3 is a lectin largely secreted by macrophages that promotes inflammation and may also directly cause insulin resistance in muscle, liver, and fat cells by interfering with insulin receptor signaling (35). Cytokines such as TNF- $\alpha$ that are locally secreted from M1 proinflammatory adipose tissue macrophages initiate NF- $\mathrm{B}$ and JNK pathways. As mentioned above, PRRs that sense endogenous ligands induced under obesogenic conditions may also play a key role, as TLR4-deficient mice are protected from the inflammatory activation induced by obesity (15). In addition, the NLR family of PRRs activates the cryopyrin/ NLRP3 inflammasome to induce IL-1 $\beta$ and IL-18 production via caspase-1 in macrophages (36). The downstream effects of PRR activation may also include regulation of inflammatory intracellular lipid species such as ceramides and sphingolipids. Inhibition of ceramide production blocks the ability of saturated fatty acids to induce insulin resistance (28). Huaizhu Wu and Christie Ballantyne discuss how the mechanisms described above mediate interactions between immune cells, adipose tissue, and skeletal muscle to influence whole-body glucose homeostasis (37).

Many of these obesity-generated inflammatory signals converge to activate serine kinases that directly block insulin action. JNK and perhaps other stress-activated kinases can inhibit insulin receptor signaling by inducing serine/threonine phosphorylation of insulin receptor substrates. This causes reduced tyrosine phosphorylation, which attenuates downstream signals (38). Furthermore, ER stress and activation of the molecular pathways governing the unfolded protein response (UPR) are closely tied to both the JNK1 and NF- $\mathrm{BB}$ pathways (39). Widespread activation of ER stress signaling components and downstream signaling cascades occurs in obesity, and pharmacologic inhibition of ER stress can reverse metabolic dysfunction in different tissues, including liver, adipose, and brain. Due to the mounting evidence supporting the roles of these pathways in obesity and related conditions, therapeutics targeting inflammation have begun to be tested in clinical trials. Allison Goldfine and Steven Shoelson review the clinical data supporting the link between inflammation and obesity and report on the outcomes of clinical trials of therapeutic strategies targeting inflammation (40).

\section{Inflammation can contribute to tissue remodeling as an adaptive response to overnutrition}

While the overall degree of inflammation correlates well with the severity of insulin resistance at steady state, inflammation may also play a part in the adaptive responses to overnutrition. These responses can involve stimulating angiogenesis to counteract hypoxia and promoting insulin resistance to protect the fat cell from overaccumulation of lipids. The expansion of adipose tissue prevents ectopic lipid deposition in other tissues such as liver and muscle, where it has lipotoxic effects (20). Healthy adipose tissue expansion requires both adipogenesis and angiogenesis to create space and support the nutrient needs of newly formed adipocytes. Endothelial cells support expansion by enabling the exchange of nutrients between the blood/lymph and tissue. Angiogenesis mediates the delivery of nutrients and oxygen to support lipid storage in adipocytes, as well as the mobilization and distribution of lipids released from adipocytes during lipolysis. Additionally, newly recruited immune cells must also cross the endothelial barrier to enter the adipose tissue. Thus, during the early phases of obesity, inflammatory processes may play an important role in supporting this adaptive response; however, continued unresolved inflammation, angiogenesis, and adipose tissue expansion ultimately leads to insulin resistance, fibrosis, adipocyte dysfunction, and cell death (20). Philipp Scherer and colleagues review the interactions between inflammation, angiogenesis, and fibrosis in the context of adipose tissue expansion (41).

\section{Conclusions}

Although the nature of the inflammatory response to obesity is unique, it does share some characteristics with other chronic inflammatory diseases, suggesting that there are lessons to be learned from such comparisons. It is likely that the initial trigger of metabolic inflammation is the disruption of energy homeostasis produced by a positive energy balance, and that the initial response is an adaptive one designed to relieve the anabolic pressure produced by obesity. However, over time this adaptive response morphs into a maladaptive one, and the sustained nature of inflammation indicates a failure to resolve the initial response to the insult. The fact that so many organ systems are impacted by inflammation during obesity suggests that new therapeutic approaches may emerge as a consequence of a better understanding of this process. Thus, advances in this field will depend critically on achieving a better appreciation of the unique and shared aspects of obesity-induced inflammation, and its intersection with the many regulatory processes that govern energy metabolism.

\section{Acknowledgments}

The authors acknowledge the support of UCSD/UCLA NIDDK Diabetes Research Center P30 DK063491.

Address correspondence to: Alan R. Saltiel or Jerrold M. Olefsky, Department of Medicine, University of California, San Diego, La Jolla, California, USA 92093. Phone: 858.534.6651; E-mail: asaltiel@ucsd. edu (A.R. Saltiel).E-mail: jolefsky@ucsd.edu (J.M. Olefsky).
1. Swinburn BA, et al. The global obesity pandemic: shaped by global drivers and local environments. Lancet. 2011;378(9793):804-814.

2. Hotamisligil GS. Inflammation and metabolic disorders. Nature. 2006;444(7121):860-867.

3. Lumeng CN, Saltiel AR. Inflammatory links between obesity and metabolic disease. J Clin Invest. 2011;121(6):2111-2117.

4. Lackey DE, Olefsky JM. Regulation of metabo- lism by the innate immune system. Nat Rev Endocrinol. 2016;12(1):15-28.

5. Lumeng CN, Bodzin JL, Saltiel AR. Obesity induces a phenotypic switch in adipose tissue macrophage polarization. J Clin Invest. 2007;117(1):175-184.

6. McLaughlin T, Ackerman SE, Shen L, Engleman E. Role of innate and adaptive immunity in obesity-associated metabolic disease. JClin Invest. 2017;127(1):5-13.
7. Eguchi K, Nagia R. Islet inflammation in type 2 diabetes and physiology. J Clin Invest. 2017;127(1):14-23.

8. Lanthier N, Molendi-Coste O, Horsmans Y, van Rooijen N, Cani PD, Leclercq IA. Kupffer cell activation is a causal factor for hepatic insulin resistance. Am J Physiol Gastrointest Liver Physiol. 2010;298(1):G107-G116.

9. Obstfeld AE, et al. C-C chemokine receptor 2 
(CCR2) regulates the hepatic recruitment of myeloid cells that promote obesity-induced hepatic steatosis. Diabetes. 2010;59(4):916-925.

10. Koyama Y, Brenner DA. Liver inflammation and fibrosis. J Clin Invest. 2017;127(1):55-64.

11. Singer K, Lumeng CN. The initiation of metabolic inflammation in childhood obesity. JClin Invest. 2017;127(1):65-73.

12. Mathis D. Immunological goings-on in visceral adipose tissue. Cell Metab. 2013;17(6):851-859.

13. Blackburn $P$, et al. Postprandial variations of plasma inflammatory markers in abdominally obese men. Obesity (Silver Spring). 2006;14(10):1747-1754.

14. Amar J, et al. Intestinal mucosal adherence and translocation of commensal bacteria at the early onset of type 2 diabetes: molecular mechanisms and probiotic treatment. EMBO Mol Med 2011;3(9):559-572.

15. Shi H, Kokoeva MV, Inouye K, Tzameli I, Yin $\mathrm{H}$, Flier JS. TLR4 links innate immunity and fatty acid-induced insulin resistance. J Clin Invest. 2006;116(11):3015-3025.

16. Winer DA, Winer S, Dranse HJ, Lam TKT. Immunologic impact of the intestine in metabolic disease. JClin Invest. 2017;127(1):33-42.

17. Oh DY, Olefsky JM. Omega 3 fatty acids and GPR120. Cell Metab. 2012;15(5):564-565.

18. Lee YS, et al. Increased adipocyte O 2 consumption triggers HIF- $1 \alpha$, causing inflammation and insulin resistance in obesity. Cell. 2014;157(6):1339-1352.

19. Trayhurn P. Hypoxia and adipose tissue function and dysfunction in obesity. Physiol Rev. 2013;93(1):1-21.

20. Sun K, Kusminski CM, Scherer PE. Adipose tissue remodeling and obesity. JClin Invest.
2011;121(6):2094-2101.

21. Khan T, et al. Metabolic dysregulation and adipose tissue fibrosis: role of collagen VI. Mol Cell Biol. 2009;29(6):1575-1591.

22. Chun TH, Hotary KB, Sabeh F, Saltiel AR, Allen ED, Weiss SJ. A pericellular collagenase directs the 3-dimensional development of white adipose tissue. Cell. 2006;125(3):577-591.

23. Jais A, Brüning JC. Hypothalamic inflammation in obesity and metabolic disease. JClin Invest. 2017;127(1):24-32.

24. Arkan MC, et al. IKK-beta links inflammation to obesity-induced insulin resistance. Nat Med 2005;11(2):191-198

25. Hirosumi J, et al. A central role for JNK in obesity and insulin resistance. Nature. 2002;420(6913):333-336

26. Nakamura T, et al. Double-stranded RNAdependent protein kinase links pathogen sensing with stress and metabolic homeostasis. Cell. 2010;140(3):338-348

27. Hotamisligil GS. Endoplasmic reticulum stress and the inflammatory basis of metabolic disease. Cell. 2010;140(6):900-917.

28. Summers SA. Sphingolipids and insulin resistance: the five Ws. Curr Opin Lipidol. 2010;21(2):128-135

29. Vandanmagsar B, et al. The NLRP3 inflammasome instigates obesity-induced inflammation and insulin resistance. Nat Med. 2011;17(2):179-188.

30. Saberi M, et al. Hematopoietic cell-specific deletion of toll-like receptor 4 ameliorates hepatic and adipose tissue insulin resistance in high-fatfed mice. Cell Metab. 2009;10(5):419-429.

31. Wellen KE, et al. Coordinated regulation of nutrient and inflammatory responses by STAMP2 is essential for metabolic homeostasis. Cell. 2007;129(3):537-548.

32. Lesniewski LA, et al. Bone marrow-specific Cap gene deletion protects against high-fat diet-induced insulin resistance. Nat Med. 2007;13(4):455-462.

33. Holland WL, et al. Inhibition of ceramide synthesis ameliorates glucocorticoid-, saturated-fat-, and obesity-induced insulin resistance. Cell Metab. 2007;5(3):167-179.

34. Li P, et al. LTB4 promotes insulin resistance in obese mice by acting on macrophages, hepatocytes and myocytes. Nat Med. 2015;21(3):239-247.

35. Li P, et al. Hematopoietic-derived galectin-3 causes cellular and systemic insulin resistance. Cell. 2016;167(4):973-984.e12.

36. Stienstra R, et al. Inflammasome is a central player in the induction of obesity and insulin resistance. Proc Natl Acad Sci U S A. 2011;108(37):15324-15329.

37. Wu H, Ballantyne CM. Skeletal muscle inflammation and insulin resistance in obesity. J Clin Invest. 2017;127(1):43-54

38. Zick Y. Ser/Thr phosphorylation of IRS proteins: a molecular basis for insulin resistance. Sci STKE. 2005;2005(268):pe4

39. Hotamisligil GS. Endoplasmic reticulum stress and atherosclerosis. Nat Med.2010;16(4):396-399.

40. Goldfine AB, Shoelson SE. Therapeutic approaches targeting inflammation for diabetes and associated cardiovascular risk. JClin Invest. 2017;127(1):83-93.

41. Crewe C, An YA, Scherer PE. The ominous triad of adipose tissue dysfunction: inflammation, fibrosis, and impaired angiogenesis. JClin Invest. 2017;127(1):74-82. 